Received 02/02/2022

Review began 02/08/2022

Review ended 02/18/2022

Published 02/26/2022

\section{(c) Copyright 2022}

Sawyer et al. This is an open access article distributed under the terms of the Creative Commons Attribution License CC-BY 4.0., which permits unrestricted use, distribution, and reproduction in any medium, provided the original author and source are credited.

\title{
The Global Healthcare Simulation Economy: A Scoping Review
}

\author{
Taylor Sawyer ${ }^{1,2}$, Megan M. Gray ${ }^{3}$, Rachel Umoren ${ }^{2}$ \\ 1. Pediatrics, Seattle Children's Hospital, Seattle, USA 2. Pediatrics, University of Washington School of Medicine, \\ Seattle, USA 3. Neonatology, Seattle Children's Hospital, Seattle, USA
}

Corresponding author: Taylor Sawyer, tlsawyer@uw.edu

\begin{abstract}
Healthcare simulation is a technique that creates a situation or environment that allows persons to experience a representation of a real health care event for the purpose of practice, learning, evaluation, or gaining an understanding of systems or human actions. The use of healthcare simulation has grown rapidly over the last decade. In this review, we describe the global healthcare simulation economy. We reviewed the literature describing the global healthcare simulation economy using four research databases (Google Scholar, MEDLINE, Embase, and EconLit) as well as alternative sources. The specific aims were to examine the major economic themes facing the healthcare simulation economy. We found that the global healthcare simulation market is segmented based on product \& services, fidelity, end-user, and geography. The market has experienced new player entry over the last few years, with most businesses focused in North America, Europe, and Asia-Pacific. The global healthcare simulation market is expected to reach between $\$ 3.19$ and $\$ 7.7$ billion by 2027 , with a compound annual growth rate of $14.6 \%$ to $17.8 \%$. Political and trade issues between America and China may increase the cost of goods in the short term. There are no global regulations on the use of healthcare simulation for training, licensing, or certification. Therefore, individual countries, states, and healthcare specialties establish individual regulations. We conclude that the major economic issues facing the global healthcare simulation economy include market segmentation, the entry of new players, and differential global growth. These factors, plus recent political and trade issues, and lack of regulations, could impact decision-making.
\end{abstract}

Categories: Medical Education, Medical Simulation, Healthcare Technology

Keywords: scoping review, economy, simulation, healthcare, global

\section{Introduction And Background}

Healthcare simulation is a technique that creates a situation or environment that allows persons to experience a representation of a real health care event for the purpose of practice, learning, evaluation, or gaining an understanding of systems or human actions [1]. Simply put, healthcare simulation is the experiential learning every healthcare professional needs but cannot engage in during real-life patient care. The use of simulation is associated with improvements in healthcare provider knowledge and skills [2]. Participation in healthcare simulation is also associated with improvements in clinical care and patient outcomes $[3,4]$.

Based on its proven benefits, the use of healthcare simulation products and services has grown rapidly over the last decade. This has caused a global expansion in both the demand and supply of healthcare simulation products and services. Understanding how the global healthcare simulation market's expansion has impacted healthcare simulation economics is essential for decision-makers. However, healthcare simulation's global economics and its impact on business decision-making have not been examined in detail.

In this research paper, we sought to describe the global healthcare simulation economy. To examine the topic, we performed a scoping review of the literature. Our specific aims were to examine the major economic issues facing the healthcare simulation economy and to investigate the influence of political, legal, regulatory, and technological issues. Based on this data, we explore how those issues impact decisionmaking by key stakeholders.

\section{Review}

\section{Search strategy}

We created a search protocol using the Preferred Reporting Items for Systematic Reviews and Meta-analysis extension for Scoping Reviews (PRISMA-ScR) [5]. We searched the medical and business literature using search terms germane to the global healthcare simulation economy. The search strategy included the following keywords and their Boolean operators: healthcare, simulation, economy, and global. The search string used was ('healthcare'/exp OR healthcare) AND ('simulation'/exp OR simulation) AND ('economy'/exp OR economy) AND ('global'/exp OR global). The search was restricted to English language publications from 
1 January 2000 to 28 February 2021. The search included four research databases: Google Scholar, MEDLINE, Embase, and EconLit. The search included examining source article citation and 'gray literature,' such as internet pages, market economic projections, corporate websites, meeting abstracts, and blogs.

\section{Synthesis of results}

Two authors screened all source content titles and abstracts for inclusion. The article's abstract was evaluated for quality and fit with the research aims for titles that seemed worthy of inclusion. If the article was applicable, qualitative and quantitative data was extracted, and then the content was analyzed and categorized [6]. Information and content from the selected publications were then categorized and grouped into major themes. When available, relevant numeric data was tabulated or presented in graphic format for easier interpretation.

\section{Results}

We identified 1,161 articles through database searching and from other sources. Of those, 19 directly related to our research aims and were included in either the qualitative or quantitative analysis (Figure 1). After a thorough review of the identified reports, we identified six major themes. The themes included 1) healthcare simulation stakeholders, 2) industries involved in healthcare simulation, 3) healthcare simulation market sectors, 4) healthcare simulation economic growth, 5) international political and trade issues, and 6) regulatory issues.

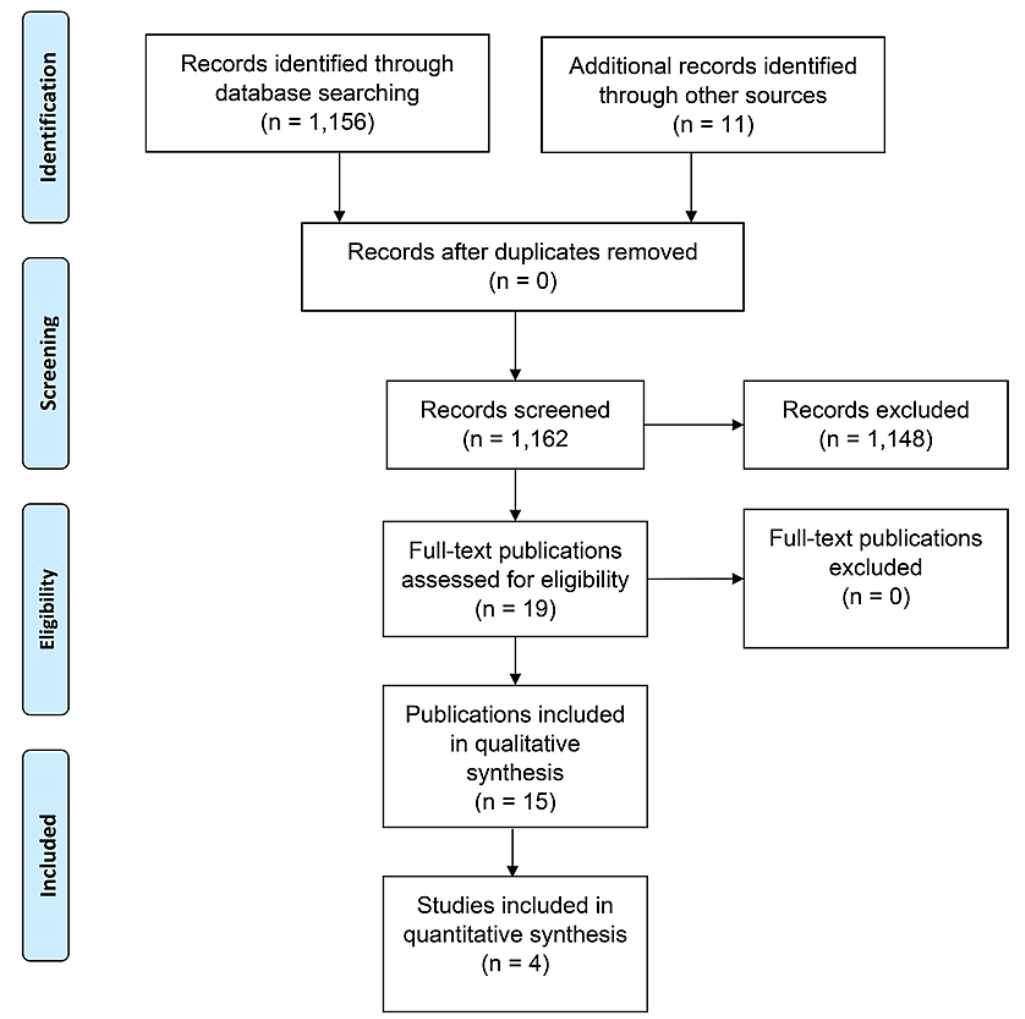

FIGURE 1: Study flow diagram

\section{Healthcare simulation stakeholders}

As part of the Research Into Global Healthcare Tools (RIGHT) project, Brailsford et al. developed a comprehensive list of potential healthcare simulation stakeholders [7]. Although the stakeholders' list focused on the U.K. National Health Service, the roles could be extrapolated outside the U.K. system and applied globally. A list of healthcare simulation stakeholders adapted from those identified by Brailsford et al. [7] is provided in Table 1 . 


\section{Cureus}

\begin{tabular}{|l|l|}
\hline Category & Example \\
\hline Government & Health ministries, Department of Education, Department of Trade \& Industry, Treasury \\
Civil services & Social care, Medicare, Medicaid \\
Public providers & National Health Service, Veterans Administration \\
Private providers & Private practice hospital and providers \\
Professional groups & British Medical Association, American Medical Association, Royal College \\
Professionals & Physicians, nurses, respiratory therapists, etc. \\
Healthcare users & Patients, families, caregivers \\
The public & Taxpayers \\
\hline
\end{tabular}

\section{TABLE 1: Healthcare Simulation Stakeholders}

Adapted from [7].

\section{Industries involved in healthcare simulation}

The Global Industry Classification Standard (GICS) is a method to assign companies to sectors, industry groups, industries, and sub-industries that best define their business [8]. The use of the GICS creates a common language among global market participants. Currently, GICS includes 11 sectors, 24 industry groups, 69 industries, and 158 sub-industries. A list of selected GICS industries involved in healthcare simulation and specific examples of products and services within the industry are shown in Table 2.

Industry*
Electrical Equipment
Professional Services
Diversified Consumer Service
Internet \& Direct Marketing Retail
Distributors
Health Care Equipment \& Supplies
Media
Electronic Equipment, Instruments \&
Components
Technology Hardware, Storage \&
Peripherals
Software

\section{Industry*}

\section{Example product and service}

Electrical components and equipment used in simulation manikins

Human resource and employment services used to hire Simulation Specialists; Healthcare simulation research and consulting services

Healthcare simulation education services and professional societies and organizations

Healthcare simulation company website and online retail space

Healthcare simulation equipment distributors

Healthcare equipment used in simulation training (e.g., gurney, stethoscope, IV equipment, etc.)

Advertising for simulation equipment and services; healthcare simulation journal and book publishing

Electronic components and instruments used in simulation manikins

Audiovisual hardware used in a simulation center

Healthcare simulation application software; software systems used to run Healthcare simulation applications

\section{TABLE 2: Industries in Healthcare Simulation}

* Global Industry Classification Standard industries [8].

\section{Healthcare simulation market segmentation}

The global healthcare simulation market can be segmented in several ways. In a 2020 report, Verified Market Research segmented the global healthcare simulation market into products and services, end-users, geography, and key players [9]. A report from Business Insights in 2020 also included the segment of end- 


\section{Cureus}

users [10]. Based on these sources, the global healthcare simulation market can be sub-segmented within these five broad categories, as seen in Table 3 .

\begin{tabular}{|c|c|}
\hline Segment & Sub-segments \\
\hline \multirow{4}{*}{ Product and service } & Healthcare anatomical models \\
\hline & Web-based simulation \\
\hline & Healthcare simulation software \\
\hline & Simulation training services \\
\hline \multirow{3}{*}{ Fidelity } & Low-fidelity \\
\hline & Medium-fidelity \\
\hline & High-fidelity \\
\hline \multirow{4}{*}{ End-user } & Academic institutions \\
\hline & Hospitals \& clinics \\
\hline & Military organizations \\
\hline & Others \\
\hline \multirow{6}{*}{ Geography } & North America \\
\hline & Europe \\
\hline & Asia-Pacific \\
\hline & Latin America \\
\hline & Middle East \\
\hline & Africa \\
\hline \multirow{6}{*}{ Key Players* } & Laerdal Medical \\
\hline & Canadian Aviation Electronics, Ltd \\
\hline & Gaumard Scientific Company, Inc. \\
\hline & Surgical Science Sweden AB \\
\hline & 3D Systems, Inc. \\
\hline & Limbs \& Things, Ltd. \\
\hline
\end{tabular}

TABLE 3: Healthcare Simulation Market Segmentation

*Select companies based on market share. Adapted from [9] and [10].

\section{Healthcare simulation economic growth}

Simulation is a small part of the healthcare field. The GICS does not list healthcare simulation as an industry or sub-industry within the "Health Care" sector [8]. However, the increasing focus on patient safety in the healthcare field is expected to boost the healthcare simulation market's growth over the next decade [9]. In recent years, the healthcare simulation market has seen the entry of many new players. In this market composed of many small and medium players, the competition is high [11].

In 2019, the global healthcare simulation market was estimated at $\$ 1.42$ to 2.27 billion [9]. The market is projected to reach somewhere between $\$ 3.19$ and $\$ 7.7$ billion by 2027, with a compound annual growth rate (CAGR) of $14.6 \%$ to $17.8 \%$ from 2020 to 2027 [9, 12]. Figure 2 provides a graphic representation of healthcare simulation growth in the U.S. from 2014 projected to 2026 [13]. 


\section{Cureus}

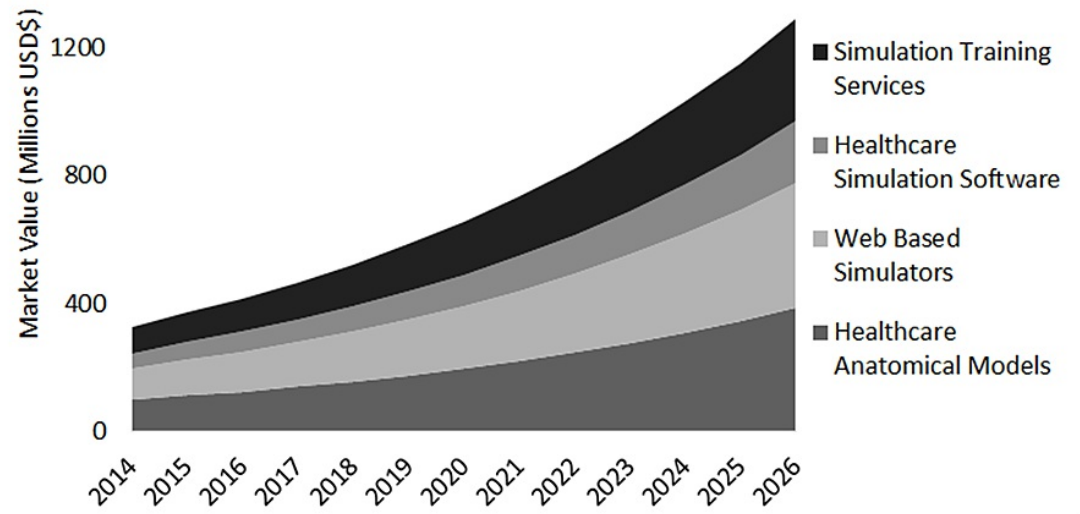

FIGURE 2: Projected healthcare simulation growth in the U.S.: 2014 to 2026

Adapted from [13].

In a market analysis, Grand View Research found that in the healthcare simulation market segment of Product \& Service, the model-based sub-segment generated the highest revenue in 2019 of $\$ 727.47$ million [14]. However, the web-based simulation segment was expected to see the highest compound annual growth rate (CAGR) of $15.2 \%$ during the period from 2020 to 2027 [14]. The low-fidelity simulators segment had the highest market share in 2019 , with $\$ 671.3$ million in the fidelity segment. However, the high-fidelity segment is expected to see the highest CAGR of 15.3\% from 2020 to 2027 [9]. As seen in Table 3, the global medical simulation market is spread across various regions. In 2019, North America accounted for nearly half of the market [10]. From 2020 to 2027, the Asia-Pacific market is expected to see the highest CAGR of $15.2 \%$ [10]. Regarding end-users, hospitals comprised more than half the market in 2019 with \$761.4 million in spending; however, academic institutions are projected to have the highest CAGR from 2020 to 2027 at $15.3 \%[10]$.

Despite the rapid growth, there are several barriers to the use of simulation globally. In the developed world, barriers to healthcare simulation adoption include lack of funding, lack of time to participate in simulation exercises, and lack of management interest or buy-in [11]. Rishipathak et al. identified the lack of capital investment as a critical barrier to the use of healthcare simulation in the developing world [15]. Rishipathak et al. speculated that in resource-limited countries 'Hub and Spoke' model - wherein one simulation center in a defined geographical area caters to all healthcare institutes in its radius - could reduce costs [15]. Young et al. identified three challenges for simulation in healthcare. The barriers included: "how good is good enough," how is modeling linked to the decision-making, and cultural barriers [16]. The cultural barriers to simulation vary from country to country.

\section{International political and trade issues}

As a global industry, healthcare simulation is impacted by international political and trade disputes. In recent years one trade issue that has impacted healthcare simulation is the U.S. and China trade war. The U.S. is the world's largest medical technology importer. China is the world's fourth-largest exporter [17]. In June 2019, the U.S. Trade Representative released a list of imports from China subject to a 25 percent tariff under Section 301 [18]. In total, the Section 301 tariffs impacted roughly $\$ 50$ billion of Chinese imports to the U.S. each year and directly impacted nearly $\$ 1.8$ billion of medical imports [19]. Those tariffs are anticipated to create a ripple effect that will increase costs in the U.S. healthcare systems. Varas and O'Neill Hayes estimated the tariffs would translate to price increases for medical equipment by roughly $\$ 400$ million in the U.S. [19]. Fernandez estimated the tariffs plus the duties on imported steel and aluminum could result in a $\$ 600$ to $\$ 800$ million increase in costs for the healthcare industry [17]. According to Varas and O'Neill Hayes, both healthcare practitioners and consumers could shoulder the increased costs in acquiring medical equipment from China [19]. The direct impact of the Section 301 tariffs on healthcare simulation costs is unclear.

\section{Regulatory issues}

Simulation is widely used in certification programs, licensing, and the assessment for credentialing in medicine, nursing, and dentistry [20]. However, there are no global regulations on the use of healthcare simulation for certification, licensing, or credentialing. Therefore, simulation is regulated independently by 
each country and regions/states within the country. However, many countries' regulatory systems can pose a substantial barrier to the consistent use of simulation [20]. For example, in the U.S., there are 70 state medical boards and 24 specialty certification boards involved in physician certification.

In the U.S., some state Boards of Nursing (BONs) allow simulation to substitute for traditional clinical learning hours for nursing students. Bradley et al. found a significant amount of variability in the number of traditional clinical hours that can be replaced with simulation in prelicensure nursing education programs [21]. The authors felt that the variability in how state BONs define and regulate simulation in nurse training raises questions about learner outcomes' consistency [21]. A meta-narrative review by Roberts et al. found that while simulation is widely used as an adjunct to clinical hours, regulations need to be defined around the simulation modality used, the number of hours of simulation compared with clinical practice, and the assessment tools used to ensure quality [22].

For physicians, the American College of Surgery and the Association for Surgical Education have developed a simulation-based medical student surgical skills curriculum for medical school students [20]. Many American Board of Medical Specialties maintenance of certification (MOC) programs use simulation as part of the MOC process. Some examples include the American Board of Anesthesiology, the American Board of Internal Medicine, and the American Board of Family Medicine.

The U.K.'s National Health Service is developing a national strategy to ensure equity of access to healthcare simulation education and training across England [23]. The goal is to ensure healthcare simulation education in the U.K. provides value and delivers high-quality and patient-centered educational outcomes [23]. Creating national regulations and standards for the delivery of healthcare simulation education and training is part of that work [24].

\section{Discussion}

The results of this scoping review revealed several key themes about the global healthcare simulation economy. Healthcare simulation is a growth industry, and economic forces favor healthcare simulation producers and service providers in the short term. The web-based simulation and high-fidelity simulation market segments are projected to have the highest near-term growth within this growing economic sector. The Asia-Pacific market and academic institutions are predicted to have the highest short-term future demand. Recent trade disputes between the U.S. and China may increase healthcare simulation equipment costs in the U.S.; however, the overall impact is unclear. A lack of global regulation around healthcare simulation requires country and state-specific knowledge of the use of healthcare simulation for training, certification, and licensure.

There are several ways this data informs decision-making. Firstly, for producers of healthcare simulation goods and services, the data reported here provide evidence that future demand will be high. This anticipated high demand justifies the heavy near-term investment of resources in this sector. Market segmentation and differential global growth must be adjusted for, however, when making decisions on where to invest resources to reap the most gains. Secondly, for healthcare simulation goods and services consumers, the expected increase in supply should bring down overall costs. This anticipated drop in costs is potentiated by the rapid entry of new players into the healthcare simulation marketplace and the overall trend for decreasing technology costs over time. Therefore, future market cost considerations are important when considering the timing of significant monetary investments in healthcare simulation. Additionally, the recent international trade disputes between the U.S. and China could increase costs within the U.S. in the short term. Finally, knowledge of regulatory issues around healthcare simulation for training, licensure, and certification is vital for both producers and consumers. Local, state, or national regulations requiring healthcare simulation use would provide an incentive towards local market growth. On the contrary, international regulations could foster growth in areas not currently invested in healthcare simulation.

This report has some limitations. Like any literature review, the results presented are entirely dependent on the search performed. We tried to perform a systematic search of the literature using published methods and applicable search terms. However, many of the final reports used in this paper were identified through other sources and within the gray literature. It is possible that another researcher using different search terms and methods may arrive at different results. Also, we did not have a method to individually verify the financial data on current market size and projected future growth. Therefore, the accuracy of that data cannot be fully validated. We included professional simulation societies and trade organizations as part of the "Diversified Consumer Service” industry (Table 2). Such organizations - which include the Society for Simulation in Healthcare (SSH), the Society in Europe for Simulation Applied to Medicine (SESAM), the International Nursing Association of Clinical and Simulation Learning (INACSL), the International Pediatric Simulation Society (IPSS), and others - collect dues from members and provide services including educational courses, individual certification, and program accreditation. Given the important roles of these professional societies in the global healthcare economy, they may warrant further investigation. Finally, the major themes noted here are based on our interpretation and analysis of the reports identified. It is possible that other researchers could have identified different or additional themes. 


\section{Conclusions}

In conclusion, to summarize our findings, the major economic issues facing the global healthcare simulation economy include diverse stakeholders, market segmentation, the entry of new players, and rapid differential global growth. In addition to these factors, recent international political and trade disputes and changing regulations around healthcare simulation could impact business decision-making by key stakeholders.

This work is important to the field of healthcare simulation because the data presented here can help inform decision-making by both suppliers and consumers. Future research in the area of healthcare simulation economics is needed to more closely examine the market forces at play within individual countries. Also, further investigation into the impact of professional healthcare simulation societies may be helpful to fully understand their impact on a national and global scale.

\section{Additional Information \\ Disclosures}

Conflicts of interest: In compliance with the ICMJE uniform disclosure form, all authors declare the following: Payment/services info: All authors have declared that no financial support was received from any organization for the submitted work. Financial relationships: All authors have declared that they have no financial relationships at present or within the previous three years with any organizations that might have an interest in the submitted work. Other relationships: All authors have declared that there are no other relationships or activities that could appear to have influenced the submitted work.

\section{Acknowledgements}

The authors would like to thank Dr. Steve Holland, Professor Emeritus, University of Washington Bothell School of Business for his input and feedback on this report.

\section{References}

1. Lioce L, Lopreiato JO, Downing D, et al.: Healthcare Simulation Dictionary. Agency for Healthcare Research and Quality, Rockville; 2020.

2. Cook DA, Hatala R, Brydges R, et al.: Technology-enhanced simulation for health professions education: a systematic review and meta-analysis. JAMA. 2011, 306:978-988. 10.1001/jama.2011.1234

3. McGaghie WC, Draycott TJ, Dunn WF, Lopez CM, Stefanidis D: Evaluating the impact of simulation on translational patient outcomes. Simul Healthc. 2011, 6:S42-S47. 10.1097/SIH.0b013e318222fde9

4. McGaghie WC, Issenberg SB, Petrusa ER, Scalese RJ: A critical review of simulation-based medical education research: 2003-2009. Med Educ. 2010, 44:50-63. 10.1111/j.1365-2923.2009.03547.x

5. Tricco AC, Lillie E, Zarin W, et al.: PRISMA extension for scoping reviews (PRISMA-ScR): checklist and explanation. Ann Intern Med. 2018, 169:467-473. 10.7326/M18-0850

6. Templier M, Paré G: A framework for guiding and evaluating literature reviews . Commun Assoc Inf Syst. 2015, 37:112-137. 10.17705/1CAIS.03706

7. Brailsford SC, Bolt T, Connell C, Klein JH, Patel B: Stakeholder engagement in health care simulation. Proc Winter Simul Conf (WSC). 2009, 1840-1849. 10.1109/WSC.2009.5429190

8. MSCI: Global Industry Classification Standard (GICS ${ }^{\circledR}$ ) methodology . (2020). Accessed: February 10, 2021: https://www.msci.com/documents/1296102/11185224/GICS+Methodology+2020.pdf/9caadd09-790d-3d60455b-2a1ed5d1e48c?t=1578....

9. Global healthcare/medical simulation market size by products \& services (healthcare anatomical models, web-based simulators, healthcare simulation software, and simulation training services), by end-users (academic institutes, hospitals, military organizations), by geographic scope and forecast. (2020). Accessed: February 9, 2021: https://www.verifiedmarketresearch.com/product/global-healthcare-medical-simulationmarket-size-and-forecast-to-2025/..

10. Allied Market Research: Medical simulation market by product \& service (model-based simulation, webbased simulation, and simulation training services), fidelity (low fidelity, medium fidelity, and high fidelity), and end user (academic institutions, hospitals, and military organizations): global opportunity analysis and industry forecast, 2021-2030. (2021). Accessed: February 10, 2021: https://www.alliedmarketresearch.com/medical-simulation-market.

11. MarketsandMarkets Research: Healthcare/medical simulation market worth $\$ 3.4$ billion by 2026 . (2020). Accessed: February 9, 2021: https://www.marketsandmarkets.com/PressReleases/healthcare-medicalsimulation.asp.

12. Allied Market Research: Medical simulation market to garner $\$ 3.19$ billion, with double-digit of CAGR 14.6\% (2020). Accessed: February 9, 2021: https://www.globenewswire.com/en/newsrelease/2020/09/10/2091513/0/en/Medical-Simulation-Market-to-Garner-3-19-Billio....

13. Grand View Research: Medical simulation market size, share \& trends analysis report by product \& services (medical simulation software, web-based simulators, simulation training services), by technology, by end use, and segment forecasts, 2021 - 2028. (2021). Accessed: February 10, 2021: https://www.grandviewresearch.com/industry-analysis/medical-healthcare-simulation-market.

14. MarketResearch.com: Medical simulation market by product \& services (model-based simulation, web-based simulation, and simulation training services), fidelity (low-fidelity, medium-fidelity, and high-fidelity), and end user (academic institutions \& research centers, hospitals. (2020). Accessed: February 20, 2021: https://www.marketresearch.com/Allied-Market-Research-v4029/Medical-Simulation-Product-ServicesModel-13689414/. 


\section{Cureus}

15. Rishipathak P, Bhandari M, Hinduja A: Simulation based healthcare education-barriers in initiation . Indian J Forensic Med Toxicol. 2020, 14:3688-3691. 10.37506/ijfmt.v14i4.12203

16. Young T, Eatock J, Jahangirian M, Naseer A, Lilford R: Three critical challenges for modeling and simulation in healthcare. Proc - Winter Simul Conf (WSC). 2009, 1823-1830. 10.1109/WSC.2009.5429186

17. US-China tariff war: what are the implications for the healthcare industry? . (2019). Accessed: March 8, 2021: https://ww2.frost.com/frost-perspectives/us-china-tariff-war-what-are-the-implications-for-thehealthcare-industry/..

18. China section 301-tariff actions and exclusion process. Office of the United States Trade Representative . (2020). Accessed: March 8, 2021: https://ustr.gov/issue-areas/enforcement/section-301investigations/tariff-actions.

19. Varas J, O'Neill Hayes T: How a Trade War with China will Impact U.S. Health Care Costs . American Action Forum, 2018.

20. Holmboe E, Rizzolo MA, Sachdeva AK, Rosenberg M, Ziv A: Simulation-based assessment and the regulation of healthcare professionals. Simul Healthc. 2011, 6:S58-S62. 10.1097/SIH.0b013e3182283bd7

21. Bradley C, Johnson B, Dreifuerst K, et al.: Regulation of simulation use in united states prelicensure nursing programs. Clin Simul Nurs. 2019, 33:17-25. 10.1016/j.ecns.2019.04.004

22. Roberts E, Kaak V, Rolley J: Simulation to replace clinical hours in nursing: a meta-narrative review . Clin Simul Nurs. 2019, 37:5-13. 10.1016/j.ecns.2019.07.003

23. Health Education England: United Kingdom National Health Service. Simulation. (2021). Accessed: March 8, 2021: https://www.hee.nhs.uk/our-work/simulation.

24. The national simulation development project: summary report. (2014). Accessed: March 8, 2021: http://aspih.org.uk/wp-content/uploads/2017/07/national-scoping-project-summary-report.pdf 